\title{
Habilidades sociales y su relación con el liderazgo en estudiantes universitarios de psicología e ingeniería
}

\author{
The role of social skills in leadership competencies on university students from psychology \\ and engineering faculties
}

\author{
Juan Tito Tenorio Romero' (iD (8)
}

\section{Cómo citar}

Tenorio Romero, J. T. (2021). Habilidades sociales y su relación con el liderazgo en estudiantes universitarios de psicología e ingeniería. Socialium, 5(2), 43-54. https://doi.org/10.26490/uncp.sl.2021.5.2.811

\begin{abstract}
${ }^{1}$ Magister en Enseñanza Estratégica, Licenciado en Pedagogía y humanidades. Universidad Continental, Huancayo, Perú.

jtenorio@continental.edu.pe
\end{abstract}

Arbitrado por pares ciegos

Recibido: 22/02/2021

Aceptado: $15 / 06 / 2021$

\section{RESUMEN}

Uno de los pendientes en el marco teórico del bienestar académico es el desarrollo del liderazgo en los estudiantes. A propósito, se han resaltado diversos aspectos de los líderes, siendo el ejercicio de las habilidades sociales uno de los que más aparece en la bibliografía. Al respecto, es importante explorarlo en población universitaria; por lo tanto, esta investigación tiene como objetivo establecer la relación entre los niveles de habilidades sociales y los niveles de liderazgo en 132 estudiantes de psicología e ingeniería en la ciudad de Huancayo, Perú. El método se baso en un estudio de tipo básico y de diseño correlacional. En los resultados, los modelos de regresión lineal muestran un efecto significativo de las habilidades sociales en la variabilidad del liderazgo de los estudiantes. $\left(R^{2}=551\right)$. De la misma forma se realizaron estadísticos comparativos para determinar el efecto específico que cada dimensión de las habilidades sociales tiene en el liderazgo total. Estos resultados permiten determinar la relación positiva que tienen las habilidades sociales en los rasgos de liderazgo en estudiantes de psicología e ingeniería de la ciudad de Huancayo - Perú. Estos mismos se consideran un aporte al desarrollo de estrategias del liderazgo académico.

Palabras clave: programa; habilidades sociales; liderazgo.

\section{ABSTRACT}

One of the pending issues in the theoretical framework of academic well-being is the development of leadership in students. By the way, various aspects of leaders have been highlighted, the exercise of social skills being one of the most appearing in the literature in this regard. It's important to explore it on university student's population. This research established the relation between soft skills and leadership levels in 132 psychology and engineering students in the city of Huancayo. On the results, linear regression model showed the significant effect of social skills on the variability of student leadership. $\left(R^{2}=551\right)$, in the same way, comparative statistics were carried out to determine the relationship effect that social skills have on total leadership $(F=34.765$; Sig. $=.000$ eta $=.525)$ as well as on the dimensions of the leadership. These results allow us to determine the positive effect that the development of soft skills had on leadership traits in psychology and engineering students from the city of Huancayo - Peru. These are considered a contribution to the development of strategies and academic leadership.

Keywords: program; social skills; leadership. 


\section{Introducción}

El estudio del liderazgo requiere, en un primer momento, determinar un concepto que nos permita orientar una investigación en este campo. Si bien existen diversas propuestas, como la clásica "Liderazgo es el logro de una meta a través de la dirección de un grupo humano. Siendo líder aquel que, de forma exitosa, orienta a sus colaboradores en el logro de algún fin particular" (Prentice, 1961). En el campo de la educación podemos abordar el concepto de liderazgo educativo, que puede tener diversas variantes tales como:

Liderazgo administrativo, basado en la aproximación tayloriana del management o la administración. Su influencia se observa actualmente en el sistema educativo por la jerarquía y burocracia. Así el líder es aquel que se enfoca en las funciones, tareas y comportamientos. Se enfatiza que esta corriente es previa al auge e integración de las ciencias sociales al estudio de la administración educativa (Bush, 2007). Liderazgo basado en el currículo, que se promueve desde la descentralización de la educación, siendo líder aquel responsable en monitorizar, implementar y mejorar la currícula escolar (Lo et al., 2012) o liderazgo docente que sugiere que los docentes tengan una posición central en la forma en la que las escuelas operen desde las funciones centrales de enseñanza y aprendizaje. Desde esta perspectiva los docentes toman parte en los mecanismos de toma de decisiones de las escuelas.

Sin embargo, el liderazgo educativo no solo se traslada a las funciones administrativas, sino a los mismos alumnos, así tenemos al liderazgo transformacional (Hallinger, 2003) teniendo cuatro componentes según Bernard Bass (Bass, The ethics of transformational leadership, 1998): Influencia idealizada, motivación inspiracional, estimulación intelectual y consideración individual. Esto permite que el liderazgo no dependa de una sola autoridad sino de múltiples recursos, lo que permite que podamos hablar también de un liderazgo estudiantil. Los líderes transformacionales resaltan por su carisma, lo que les permite comunicar una visión, motivando a sus seguidores a pensar de forma divergente, altas expectativas y confianza. Resaltando su valor y comprometiéndose a tener una conducta ejemplar y simbólica.

El enfoque transformacional, debido a su característica psicológica no deja de lado los enfoques anteriores como el transaccional, de orientación más administrativa estando basada en el intercambio de recompensas contingentes en el desempeño (Khan, 2017), es decir enfocarse en la relación líder seguidor. Aquí el líder estudiantil está llamado a aprender a dar recompensas por el buen desempeño. Al respecto se ha planteado un modelo o teoría de liderazgo (transformacional y transaccional) propuesto por Bass y Avolio (2003) y adaptado al entorno educativo por Aguilar et al. (2015) desde las investigaciones de Nader y Sánchez (2010), así como el modelo de Cárdenas (2016) y en base a la teoría de la presente investigación. Este modelo tiene básicamente tres dimensiones: 
La primera dimensión denominada consideración individual, donde se reconoce a los estudiantes por sus relaciones con los demás, siendo sociables, (lo que será la lógica principal de este estudio para su relación con las habilidades sociales) y logrando estar rodeado por personas que se sientan bien a su lado debido a la calidad de sus relaciones. Es el momento o espacio donde el individuo se involucra con sus compañeros de estudio o trabajo en diversas actividades laborales o grupales desarrollando su potencial y mostrando una consideración individualizada, que sería la primera dimensión.

La segunda dimensión, motivación inspiradora, que aborda la destreza para motivar a otras personas en el entorno para que se sientan cómodos con el trabajo de grupo; la tercera dimensión, estimulación intelectual, se muestra en momentos de crisis donde los subordinados puedan desarrollar independencia y autonomía adecuada. Finalmente, se encuentra la influencia idealizada, que evalúa el carisma; es decir, la confianza y respeto que inspiran, lo que permite poder relacionarse libremente con sus seguidores tanto para sancionarlos como para resaltar sus logros.

En una revisión sistemática, se encontró que, de 183 artículos, 12 tenían el propósito de proponer un modelo de desarrollo de liderazgo (Gumus et al., 2016). La razón podría residir en que hasta la fecha se vienen construyendo y probando diversos modelos teoréticos para poder construir programas integrales en la preparación y desarrollo de líderes, los que requieren de la ejecución de diversas actividades que exigen una logística mayor y apoyo de diversos agentes privados y gubernamentales. Sin embargo, una alternativa es seguir explorando modelos y teorías asociadas al liderazgo educativo, como la ya mencionada por Aguilar et al., (2015).

Las habilidades sociales han sido estudiadas en amplio número de ensayos en búsqueda de esfuerzos que provienen desde Vigostky, enfatizando la interacción social como la forma en la que el sujeto se desarrolla apropiándose de conocimiento (Ríos, 2009), pasando por la perspectiva social de Bandura base para diversos programas (Dextre et al., 2017) y llegando finalmente a Caballo (1987) desde cuyo esfuerzo se han propuesto componentes conductuales (no verbales, paralingüísticos o vocales, verbales y mixtos) mismos que se han explorado en diversas investigaciones (Benzer, 2012) (Gadzhiyeva y Sager, 2017) (Cárdenas y Duarte, 2016) donde se muestra el nexo emocional y social que se puede alcanzar desde la intervención de estos componentes.

Esta objetividad de abordaje de las habilidades sociales y su nexo con el modelo de liderazgo de Bass y Avolio, en las dimensiones de liderazgo transaccional y transformacional, nos lleva a considerar que se puede generar un efecto significativo, específicamente con esta última dimensión, que desde la cual se ha construido la escala, a partir de la modificación de las habilidades sociales. Lo que sería un aporte sustancial a las investigaciones para el desarrollo del liderazgo. 
Si existe un punto claro en la literatura es que el carisma personal depende de la habilidad de la persona para comunicarse, principalmente la comunicación de emociones, la habilidad de interpretar las emociones en los demás y la habilidad para controlar su comunicación emocional. Lo que ha llevado a definir al carisma como el desarrollo de la combinación de habilidades sociales y de comunicación (Akville, et al., 2018), estas personas tienen más probabilidades de ser considerados líderes y tienen redes sociales más amplias, más amigos, mayor soporte social y mejor adaptabilidad.

Una investigación (Riggio y Salinas, 2003) ha mostrado, de forma aplicada y observacional, la relación entre el rol de las habilidades sociales en la efectividad del liderazgo. Implementando grupos, en tres estudios, donde universitarios pudieron trabajar en pequeños grupos en tareas asociadas a la resolución de problemas. Los resultados mostraron que las habilidades sociales se relacionaron con la satisfacción del líder, pero solo relacionada al desempeño de liderazgo en líderes de alto nivel o jerarquía. Los mismos autores han generado un trabajo monográfico más amplio (Riggio y Tan, Leader Interpersonal and Influence Skills, 2014), otras investigaciones han relacionado ambas variables y resaltado como los contextos socio-culturales y las habilidades sociales aprendidas de estas influencias de las habilidades de liderazgo (Maricela, 2019) (Reza y Aziz, 2014).

Esto nos llevó a evaluar en un solo momento a un grupo de estudiantes tanto en habilidades sociales como en liderazgo. Resolviendo la siguiente pregunta de investigación. ¿Cuál es la relación que tienen las habilidades sociales en los niveles de liderazgo en alumnos de psicología e ingeniería de la universidad Continental de la ciudad de Huancayo?

\section{Método}

Tipo de estudio. El tipo de investigación fue básico ya que se caracteriza por la exploración de teorías previas para construir y precisar el conocimiento sobre los fenómenos o eventos abordados. El diseño básico utilizado fue el no experimental, transversal y descriptivo correlacional que según Hernández et al. (2010) Describen relaciones entre dos o más categorías, conceptos o variables en un momento determinado.

Población y muestra. El estudio fue aplicado en estudiantes universitarios de la Universidad Continental, de las facultades de psicología e ingeniería. Siguiendo un tipo de muestreo probabilístico aleatorio se logró evaluar a 132 estudiantes de estas facultades.

Instrumentos de recolección de datos. Los instrumentos utilizados fueron: La (EHS), Escala de habilidades sociales, (Gismero, 1996) cuyo Autora es Elena Gismero González de la Universidad Pontificia Comillas 
(Madrid) perteneciente a la Facultad de filosofía y letras, Sección Psicología. La administración del instrumento es Individual o colectiva. Su duración es variable de aproximadamente 10 a 15 minutos. Dirigida su aplicación a adolescentes y adultos. Con significación dirigida a la evaluación de la aserción, las habilidades sociales y tipificación en base a niveles de población general (varones y mujeres, adultos y jóvenes). Para la elaboración del instrumento de la escala de habilidades sociales ha tenido que pasar por la aplicación de tres muestras de diferentes grupos heterogéneos. El instrumento de 33 ítems tiene una alta consistencia interna como se expresa en un coeficiente de fiabilidad alto alfa $=0.88 \mathrm{El}$ instrumento EHS ha pasado por un ajuste semántico y fue adaptado al contexto social de la región ya que se aplicó a una muestra de 20 estudiantes con la finalidad de determinar su validez gramatical en los ítems obteniendo una media aritmética de 0.91 , permitiendo la aplicación a la muestra respectiva.

Y la escala MLIS, elaborada por Cárdenas (2016), la cual consta de 28 ítems que se divide en cuatro dimensiones: Influencia idealizada, motivación inspiradora, estimulación intelectual y consideración individualizada. Brindándose la respuesta a través de una escala Likert de 0 a 4 . Donde 0 es nunca, 1 casi nunca, 2 a veces, 3 casi siempre y 4 siempre. Este instrumento cuenta con validez de criterio, debido al criterio de jueces con la que fue adaptada; y el alfa de Cronbach (.92) que se obtuvo en su proceso de confiabilidad.

Procedimientos de la recolección de datos: El proceso se llevó a cabo mediante un formulario online que contuvo, tanto el consentimiento informado, como ambas escalas (Habilidades sociales y liderazgo), el mismo fue entregado durante la cátedra de Liderazgo impartida a los alumnos de estudios generales de las facultades de Psicología e ingeniería

Aspectos éticos. Todos los participantes rellenaron un consentimiento informado que contenía los objetivos de la investigación y los riesgos considerados desde la evaluación. Todos los procedimientos se llevaron tomando en cuenta la declaración de Helsinki de la AMM-Principios éticos para las investigaciones en seres humanos.

Análisis de datos. El procesamiento de los datos se hizo a través del IBM SPSS Statistics 12 con el que se realizó la prueba $r$ de Pearson para ver el nivel y grado de correlación, además de las modelaciones de regresión lineal para determinar el tamaño del efecto de esta variabilidad. Además, se llevó a cabo la ANOVA de un factor para determinar las diferencias entre cada una de las habilidades sociales según las cuatro dimensiones del liderazgo. $Y$ así reconocer entre cuales se registran diferencias significativas. Se realizaron también pruebas post-hoc para reconocer las diferencias específicas entre estas dimensiones 


\section{Resultado}

\section{Datos descriptivos}

En un primer momento se exploraron las medidas de tendencia de los niveles de liderazgo, describiéndolas según los niveles de habilidades sociales obtenidas por los evaluados. Adicionalmente llevó a cabo un proceso de análisis de varianza de medias a través del estadístico $F$ de Fisher para determinar el grado de asociación entre las dimensiones de liderazgo según los niveles de habilidades sociales. Como se observa en la tabla 1 , se encuentran diferencias estadísticamente significativas en cada una de las dimensiones.

\section{Tabla 1}

Análisis descriptivo de las dimensiones de Liderazgo según niveles de habilidades sociales

\begin{tabular}{|c|c|c|c|c|c|c|}
\hline & \multicolumn{3}{|c|}{ Habilidades Sociales } & \multicolumn{2}{|c|}{ ANOVA } & \multirow[t]{2}{*}{$\mathrm{Eta}^{2}$} \\
\hline & $\begin{array}{l}\text { Muy Alto } \\
\text { Mean (SD) }\end{array}$ & $\begin{array}{c}\text { Alto } \\
\text { Mean (SD) }\end{array}$ & $\begin{array}{l}\text { Medio a bajo } \\
\text { Mean (SD) }\end{array}$ & $F$ & P-Valué & \\
\hline $\begin{array}{l}\text { Influencia } \\
\text { idealizada }\end{array}$ & $16,30(1,82)$ & $13,50(1,45)$ & $11,82(1,32)$ & 31,215 & ,000 & ,405 \\
\hline $\begin{array}{l}\text { Motivación } \\
\text { inspiradora }\end{array}$ & $25,35(2,82)$ & $21,06(2,36)$ & $18,18(1,94)$ & 36,319 & ,000 & ,563 \\
\hline $\begin{array}{l}\text { Estimulación } \\
\text { intelectual }\end{array}$ & $16,30(1,82)$ & $13,50(1,45)$ & $11,82(1,32)$ & 36,215 & ,000 & ,535 \\
\hline $\begin{array}{l}\text { Consideración } \\
\text { individualizada }\end{array}$ & $32,57(3,69)$ & $27,09(3,16)$ & $23,36(2,61)$ & 38,991 & ,000 & 619 \\
\hline Total & $90,52(10,26)$ & $75,22(8,59)$ & $65,00(7,23)$ & 34,765 & ,000 & ,525 \\
\hline
\end{tabular}

\section{Prueba de hipótesis}

Se realizó un análisis de correlación entre las variables referidas en las hipótesis de este estudio. Los resultados de las correlaciones de Pearson se presentan en la tabla 1 y 2 . Como se observa, todas las relaciones entre las variables resultaron ser significativas $(p<0.01)$ Específicamente y basado en la interpretación standard del tamaño de efecto (Cohen, 1988) podemos ver que todas las asociaciones son altas ( $r>50)$; siendo solo la asociación entre la habilidad "Iniciar interacciones" y Liderazgo, la única que ha mostrado una asociación moderada $(\mathrm{r}=0.42)$

\section{Tabla 2}

Correlación entre las habilidades sociales y los niveles generales de liderazgo

\begin{tabular}{cc}
\hline Correlación & $\begin{array}{c}\text { Liderazgo } \\
M=62,95 \\
\end{array}$ \\
& $D=12,68$ \\
\hline Habilidades Sociales \\
$M=62,95$ & $r=742 * *$ \\
$D=12,68$ & Sig. $=, 000$ \\
\hline$* *$. La correlación es significativa en el nivel 0.01 (bilateral).
\end{tabular}


Tabla 3

Análisis de correlación entre Liderazgo, la escala total de Habilidades sociales y sub escalas

\begin{tabular}{|c|c|c|c|c|c|c|c|}
\hline & 1 & 2 & 3 & 4 & 5 & 6 & 7 \\
\hline 1. Liderazgo & - & $0.57^{* *}$ & $0.69^{* *}$ & $0.56^{* *}$ & $0,67^{* *}$ & $0,57^{* *}$ & $0,42^{* *}$ \\
\hline 2. autoexpresión de situaciones s & & - & - & - & - & - & - \\
\hline 3. Defensa de derechos propios & & & - & - & - & - & - \\
\hline 4.Expresión de enfado & & & & - & - & - & - \\
\hline 5. Decir no & & & & & - & - & - \\
\hline 6. Hacer peticiones & & & & & & - & - \\
\hline 7. iniciar interacciones & & & & & & & - \\
\hline
\end{tabular}

Adicionalmente se llevó a cabo un cuadro de dispersión, que puede observarse en la Figura 1, donde se aprecia la relación directa y el indicador de validez del modelo de regresión $R^{2}=0,551$; a través del cual podemos corroborar el efecto explicativo, alto y significativo, del liderazgo (escala total) a través de las habilidades sociales (escala total).

\section{Figura 1}

Cuadro de dispersión de liderazgo según habilidades sociales

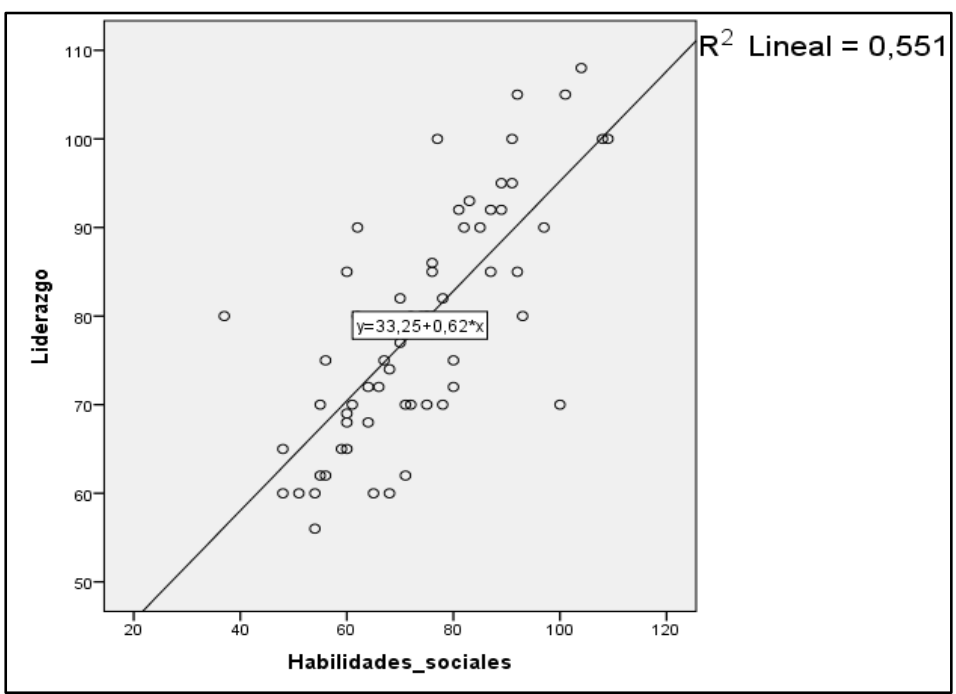




\section{Discusión}

Los resultados nos permiten corroborar la relación entre las habilidades sociales y los estilos de liderazgo, algo que ya la literatura ha sugerido en diversas investigaciones (Riggio y Salinas, 2003; Keow et al., 2015), (Gumus et al., 2016) por lo que se replican los resultados. El aporte de la presente investigación radica en el énfasis de estas habilidades con un modelo de liderazgo transformacional, el cual es de un corte más psicológico ya que enfatiza, como ya se ha mencionado, las habilidades de comunicación como, base del carisma, para motivar a los seguidores a una meta determinada.

Al respecto es resaltante que las dos habilidades sociales con mayor correlación a los niveles de este tipo de liderazgo sean "la defensa de derechos propios" ( $r=0.69^{* *}$ ) y el "decir no y cortar interacciones" ( $r=$ $0.67^{* *}$ ) que son habilidades más orientadas a la defensa de la propia identidad, y no necesariamente a la dimensión "autoexpresión emocional" que era la esperada. Esto nos permite comprender que, desde las habilidades sociales, el liderazgo transformacional se relaciona, en un primer momento, a las capacidades de defender los valores del grupo porque se tiene la habilidad de defender el derecho propio; y de poder mantener la autoridad, muchas veces, cortando interacciones que no sean congruentes a los valores del grupo, esto es acorde a la integración del modelo transformacional con el transaccional (Bass y Avolio, 2003) ; donde se sugiere que las capacidades del líder transaccional, muchas veces mal percibidas como severas o impositivas, son complementarias a la expresión de emociones que van construyendo la característica confianza hacia el líder transformacional

Se ha mostrado evidencia estadística de las diferencias de las dimensiones del liderazgo según los niveles de las habilidades sociales y gracias a las pruebas estadísticas específicas, encontramos que la variabilidad del liderazgo, según las habilidades sociales, se encuentra en mayor medida en la dimensión consideración individualizada. Es decir, que las habilidades sociales mostrarían su mayor urgencia, en un líder, al momento de incluir a las personas a un grupo gracias a sus capacidades de sociabilizar, lo que es coherente a la bibliografía ya mencionada (Bush, 2007). Luego esta variabilidad se encontraría en la dimensión "motivación inspiradora", es decir en el momento de motivarlos a la acción. El carisma, en la forma de la influencia idealizada es la que muestra menor nivel de variabilidad. Lo que sugeriría que, si bien, el líder transformacional se caracteriza por su carisma que lleva a que sus seguidores construyan un ideal de este (Akville et al., 2018), su liderazgo se basa en otros aspectos previos donde las habilidades sociales son fundamentales tanto para convocar a un grupo como para motivar a la acción. El carisma permitiría mantener este liderazgo, pero no impulsarlo al inicio.

Finalmente es importante considerar dos puntos, el primero con respecto a las limitaciones del estudio donde consideramos que la observación de las habilidades sociales y el liderazgo no se realizó mediante 
la observación de una tarea, o mediante un programa de desarrollo de habilidades sociales en empresarios, donde podría atribuirse una razón de causalidad. Sin embargo, como segundo punto, consideramos que la presente investigación apunta a la discusión del liderazgo desde una perspectiva relacional, con bases en las relaciones sociales, lo que debatiría la posibilidad de que el liderazgo sea un rasgo característico de los individuos y no algo que se va formando y retroalimentado desde la calidad relacional con otros individuos, donde se aprendería a liderar en base a las habilidades sociales en diversas situaciones propias del ejercicio de dirigir un equipo o grupo hacia una meta.

\section{Conclusión}

La presente investigación parte de la necesidad de conocer, de manera más específica, como se relacionan las habilidades sociales con el liderazgo transformacional. Los resultados aquí expuestos muestran cómo, desde las diferencias de media de los niveles de habilidades sociales, algunas dimensiones del liderazgo están más asociadas a esta variabilidad, lo que permitiría proponer un modelo de intervención desde una lógica que priorice ciertos aspectos del liderazgo transformacional, pero también del administrativo, logrando una sinergia entre ambas perspectivas. Por otra parte, los resultados permiten identificar las habilidades sociales más relacionadas con dimensiones de liderazgo transformacional y administrativo. Lo que ayudaría a proponer tareas específicas de entrenamiento en habilidades sociales y comprobar si siguen la lógica previamente mencionada, de sinergia entre ambas perspectivas. Finalmente, el presente trabajo apunta a sostener que el liderazgo se aprende básicamente en la relación efectiva con otras personas, en el marco de los objetivos de un grupo; lo que pone en debate la postura de un rasgo inherente de liderazgo en las personas.

\section{Referencias}

Aguilar, V., Burgos, A., Argueta, M., Linares, D. y Marroquin, W. (2015). Liderazgo estudiantil y cuestionario de liderazgo estudiantil. Universidad de Oriente.

Akville, V., Aurelija, Z. y Vilma, Z. (2018). Charisma as a phenomenon in social work. Vocational training: Research and Realities, 29(1), 58-69. https://doi.org/10.2478/vtrr-2018-0005

Bass, B. (1998). The ethics of transformational leadership. Ethics, the Heart of Leaders, 3(3), 169-192. https://doi.org/10.15209/jbsge.v3i3.139

Bass, B. y Avolio, B. (2003). Predicting unit performance by assessing transformational. Journal of Applied Psycology, 82(8) 207-218. https://doi.org/10.1037/0021-9010.88.2.207 
Benzer, A. (2012). Teachers opinion about the use of body language. Education, 132(3), 467-473. https://www.researchgate.net/profile/Ahmet-

Benzer/publication/346027199_Teachers\%27_opinions_about_the_use_of_body/links/5fb6c9f0 a6fdcc6cc64be7f1/Teachers-opinions-about-the-use-of-body.pdf

Bush, T. (2007). Educational leadership and management: Theory, policy and practice of education. South African journal of education, 27(3), 391-406. Recuperado de: https://wrap.warwick.ac.uk/426/

Caballo, V. (1987). Teoría, evaluación y entrenamiento de las habilidades sociales. Promolibro.

Caballo, V. (2009). Manual de evaluación y entrenamiento de las habilidades sociales (5ta. ed.). Siglo XXI.

Cárdenas, C. y Duarte, C. (2016). Proxémica, Kinésica y Antropología. Apuntes sobre simulación etnográfica, cuerpo y espacio en el marco del conflicto armado colombiano. Antípoda, (25), 3359. https://doi.org/10.7440/antipoda25.2016.02

Cárdenas, J. (2016). Construcción de instrumento para medir el liderazgo en estudiantes de secundaria [Tesis de pregrado. Pontificia Universidad Católica del Perú]. Repositorio institucional PUCP]. http://tesis.pucp.edu.pe/repositorio/handle/20.500.12404/8463

Dextre, H., Susana, J., García, L. y Yessica, C. (2017). Programa "yo con nueva actitud" basado en la Teoría de Bandura para fortalecer las habilidades sociales en los niños y niñas de segundo grado de Educacion Primaria de la Institucion Educativa "Augusto Salazar Bondy" de Nuevo Chimbote, 2015. [Tesis de pregrado, Universidad Nacional del Santa]. Repositorio institucional UNS. http://repositorio.uns.edu.pe/handle/UNS/3005

Elliot, S. y Gresham, F. (2008). SSiS: Social Skills Improvement System: Intervention Guide. Pearson.

Gadzhiyeva, N. y Sager, K. (2017). Maximizing the persuasiveness of a salesperson: An exploratory study of the effects of nonverbal immediacy and language power on the extent of persuasion. Journal of Work and Organizational Psychology, (33), 83-93. https://doi.org/10.1016/j.rpto.2017.03.001

Gismero, E. (1996). Manual de escala de Habilidades Sociales. Madrid: Universidad Pontificia Comillas.

Gumus, S., Esen, M., Sukru, M. y Gumus, E. (2016). A systematic review of studies on leadership models in educational research from 1980 to 2014. Educational management admnistration and leadership, 46(1), 25-48. https://doi.org/10.1177/1741143216659296

Hallinger, P. (2003). Leading educational change: Reflections on the practice of instructional and transformational. Cambridge Journal of Education, 33(3), 329-352.

https://doi.org/10.1080/0305764032000122005 
Hosokawa, R. y Katsura, T. (2017). A longitudinal study of socioeconomic status, family processes, and child adjustment from preschool until early elementary school: the role of social competence. Child y Adolescent Psychiatry y Mental Health, 11(63). https://doi.org/10.1186/s13034-017-0206-z

Keow, T., Huwaina, S. y Kanokorn, S. (2015). Soft Skills of leaders and school improvement in high performing schools. Procedia-Social and behavioural sciences, (191), 2127-2131. https://doi.org/10.1016/j.sbspro.2015.04.652

Khan, N. (2017). Adaptive or transactional leadership in current higher education: A brief Comparison. International review of research in open and distributed learning, 18(3), 179-183. https://doi.org/10.19173/irrodl.v18i3.3294

Lo, Y., Adamson, A. y Lam, J. (2012). Curriculum leadership in schools. Curriculum Change, 231-254. https://doi.org/10.5790/hongkong/9789888139026.003.0001

Mafra, H. (2015). Development of learning and social skills in children with learning disabilities: an educational intervention program. Procedia Social andBehavioral Sciences, (209), 221.228. https://doi.org/10.1016/j.sbspro.2015.11.220

Mantilla, B., Hakspiel, M., Guerrero, N., Niño, M. L.,Mantilla, L. y Cardenas, M. (2016). Efectividad del proceso de formación de escolares de 32 colegios públicos en habilidades psicosociales y hábitos saludables en Engativá-Bogotá D.C. Aquichan,16 (4) 462-472. https://doi.org/10.5294/aqui.2016.16.4.5.

Matson, J. (2017). Handbook of Social Behavior and Skills in Children. Autism and Child Psychopathology. Series Springer.

Nader, M., y Sanchez, E. (2010). Estudio comparativo de los valores de líderes trasnformacionales y transaccionales civiles y militares. Anales de psicología, 26(1) 72-79. https://revistas.um.es/analesps/article/view/91991

OECD. (2015). Skills for Social Progress: The Power of Social and Emotional Skills. OECD.

Prentice, W. (2004). Undestanding Leadership. Harvard Business Review, 82(1) 102-109. http://psgleadership.scripts.mit.edu/2011IAP/pdf/1_required_reading/Understanding\%20Leade rship.pdf

Pulido, F. y Herrera F. (2016). Predictores del miedo y las habilidades sociales en adolescentes del contexto pluricultural de ceuta. Acción Psicológica, 13(1) 179-191. https://doi.org/10.5944/ap.13.1.15560 
Punset, E. (2014). El mundo en tus manos. Destino.

Riggio, R. y Salinas, C. (2003). The role of social and communication skills in leader emergence and effectiveness. Group Dynamics theory research and practice, 7 (2) 83-103. https://doi.org/10.1037/1089-2699.7.2.83

Rios, V. (2009). Entrenamiento de habilidades y competencias en las organizaciones. Publimass.

Rojas, C., Guadalupe, M., Romero, M. y Sanchez, T. (2015). Relación entre funciones ejecutivas y habilidades sociales en adolescentes: un estudio piloto. Revista Intercontinental de Psicología y Educación, 17(2), 167-183. https://www.redalyc.org/articulo.oa?id=80247939009

Tenorio, J. (2016). Programa triunfadores y habilidades sociales en estudiantes de psicología e ingeniería en la ciudad de Huancayo [Tesis de Maestría, Universidad Nacional del Centro del Perú]. Repositorio institucional UNCP. http://repositorio.uncp.edu.pe/handle/20.500.12894/4481

\section{Fuentes de financiamiento}

Autofinanciada por el mismo autor de la investigación.

\section{Conflictos de interés}

No presenta ningún conflicto de interés.

\section{Correspondencia}

jtenorio@continental.edu.pe 